\title{
Erratum to: 3D Diffractive Lenses to Overcome the 3D Abbe Diffraction Limit
}

\section{Erratum to:}

I. Minin and O. Minin, Diffractive Optics

and Nanophotonics, SpringerBriefs in Physics, DOI 10.1007/978-3-319-24253-8_2

Erratum DOI: 10.1007/978-3-319-24253-8_7

The book was inadvertently published with an error in the title as "3D Diffractive Lenses to Overcome the 3D Abby Diffraction Limit", the correct title is "3D Diffractive Lenses to Overcome the 3D Abbe Diffraction Limit". 